\title{
Analyse automatique de l'écriture manuscrite en ligne pour la détection précoce des pathologies neurodégénératives
}

\author{
Automatic analysis of on-line handwriting for the early detection of
} neurodegenerative pathologies

\author{
Aouraghe Ibtissame ${ }^{1}$, Ammour Alae $^{1}$, Aboulem Ghita $^{2}$, Khaissidi Ghizlane ${ }^{1}$, Mrabti Mostafa ${ }^{1}$, \\ Belahsen Faouzi ${ }^{2}$, Mounim A. El-Yacoubi ${ }^{3}$, Sonia Garcia-Salicetti ${ }^{3}$ \\ ${ }^{1}$ Laboratoire LIPI ENS, USMBA Fès, Maroc, aouraghe.ibtissame@gmail.com, alae.am19@gmail.com, \\ ghizlane.derkaoui1@hotmail.com, mostafa.mrabti@yahoo.fr \\ ${ }^{2}$ Laboratoire ERMSC, FMPF, CHU Hassan II Fès, Maroc, aboulemghita@gmail.com, belahsenfaouzi@gmail.com \\ ${ }^{3}$ SAMOVAR, Telecom SudParis, CNRS, Université Paris Saclay, France, mounim.el_yacoubi@telecom-sudparis.eu, \\ sonia.garcia@telecom-sudparis.eu
}

RÉSUMÉ. L'objectif de cet article est d'analyser l'écriture manuscrite en ligne, caractérisée par une intervention primordiale des muscles et du cerveau, afin de détecter des pathologies neurologiques comme l'Alzheimer et le Parkinson, qui sont liées à l'organisation de l'ensemble des facultés mentales. Dans ce cadre, une acquisition de données est réalisée au sein du service neurologie au CHU Hassan II de Fès. Elle est faite sur une tablette graphique WACOM permettant de récupérer les données spatiotemporelles de l'écriture manuscrite. La problématique de la détection de pathologies nécessite préalablement l'étude des paramètres descriptifs de l'écriture manuscrite permettant la caractérisation des sujets contrôles et des personnes atteintes. Cet article décrit principalement la phase d'acquisition de données en cours au CHU de Fès, ainsi que l'analyse préliminaire de paramètres spatiotemporels de l'écriture réalisée sur ces données.

ABSTRACT. We propose in this article to analyse on-line handwriting acquired on a digital graphic tablet for the early detection of neurological pathologies. Our main goal is the characterization of neurodegenerative diseases, such as Parkinson, Alzheimer, Mild Cognitive Impairment and neuropsychiatric diseases such as schizophrenia, through the use of spatiotemporal parameters of handwriting. This paper mainly describes the phase of handwriting and hand-drawn data acquisition currently underway at the Neurological Service at CHU Hassan II de Fez hospital, and gives a preliminary analysis of the spatiotemporal parameters extracted from the handwriting.

MOTS-CLÉS. Ecriture manuscrite, tablette graphique, Parkinson, Alzheimer, Troubles cognitifs Legers.

KEYWORDS. Handwriting, graphic tablet, Parkinson, Alzheimer, mild cognitive impairment.

\section{Introduction}

L'écriture manuscrite se caractérise principalement par une intervention primordiale des muscles et du cerveau. Ceci montre à la fois la subtilité et la complexité de l'écriture, qui touche aux mécanismes les plus profonds de la sensorimotricité et de la cognition.

La dégradation de l'écriture des personnes est due principalement à des pathologies comme 1'Alzheimer, le Parkinson, le Cancer, des maladies cardiaques et des pathologies psychiatriques potentielles comme la schizophrénie.

De ce fait, l'analyse de l'écriture manuscrite est devenue un terrain d'exploration nouveau et prometteur des chercheurs dans le domaine de la santé, notamment, la neuroscience et l'étude des troubles neurologiques. Partant du fait que certaines modifications de l'écriture provenant d'un mouvement irrégulier peuvent être évocatrices d'une maladie, un texte manuscrit dégradé pourrait apporter des informations diagnostiques considérables sur son auteur, ce qui présente des intérêts variés scientifiquement et socialement exploitables. 
Les pathologies neurodégénératives comme le Parkinson, l'Alzheimer et les troubles cognitifs légers (MCI), sont souvent caractérisées par des troubles de fluidité de mouvement dans l'écriture. Plusieurs travaux de recherche ont exploité l'émergence rapide des technologies numériques pour analyser les troubles de l'écriture chez les patients atteints de ces maladies. La littérature évoque principalement l'utilisation de deux approches technologiques fondamentales pour l'analyse de l'écriture : l'une est appelée "Hors-ligne » où le manuscrit est capturé et traité en tant qu'image, tandis que la technologie dite " en-ligne » permet l'acquisition du manuscrit en temps réel à partir d'une tablette graphique qui extrait des paramètres cinématiques et spatio- temporels liés à l'interaction entre le stylet et la surface de la tablette. De fait, la majorité de ces travaux ont statistiquement prouvé, à base d'un ensemble d'exercices d'écriture subtilement élaborés, l'existence d'une forte relation entre la dégradation de l'écriture et les maladies neurodégénératives, qui pourrait être pratiquement investie pour l'aide au diagnostic et le suivi de la progression de ces pathologies.

La maladie de Parkinson, l'Alzheimer et le MCI affectent le plus souvent les individus âgés, et touche annuellement des milliers de personnes dans le monde. Or, les critères permettant de porter un diagnostic précoce et fiable de ces maladies restent limités à l'heure actuelle vu que les symptômes, aux premiers stades, peuvent passer inaperçus. Ceci explique que toute étude qui sera orientée dans ce sens aura une valeur scientifique précieuse, et tout résultat qui en découle pourrait contribuer considérablement à l'amélioration de la qualité de vie des patients. D'autre part, l'exploitation des paramètres cinématiques de l'écriture manuscrite constitue un enjeu majeur et primordial pour la détermination de l'ensemble des attributs portant l'information diagnostique de ces pathologies.

Notre projet de détection de pathologies à travers l'analyse de l'écriture manuscrite en ligne s'inscrit dans le cadre d'une collaboration entre notre laboratoire d'Informatique et de Physique Interdisciplinaire (LIPI), l'équipe Armedia du laboratoire SAMOVAR de Telecom SudParis, le service de neurologie au CHU Hassan II de Fès et l'hôpital de Broca à Paris. Toute l'équipe a montré sa motivation pour relever le défi et chercher de nouveaux moyens diagnostiques simples et innovants pour la détection précoce des pathologies neurodégénératives en se basant sur l'analyse de l'écriture manuscrite chez les deux populations : Marocaine et Française qui ont bien entendu, des aspects culturels et sociaux différents. Cette étude comprend principalement deux phases :

- Phase d'acquisition au sein du Centre Hospitalier Universitaire Hassan II à Fès permettant de construire une base de données d'une population bilingue ou monolingue.

- Phase d'extraction et d'analyse des paramètres descriptifs de l'écriture manuscrite acquise de sujets contrôles et de personnes atteintes.

Cet article a pour but de décrire exhaustivement la phase d'acquisition de données en cours, faite principalement sur la population marocaine, et sera organisé comme suit:

- Dans un premier temps, nous présenterons l'état de l'art sur l'analyse de l'écriture pour les personnes atteintes du Parkinson, de l'Alzheimer et des troubles cognitifs légers. Cette étude motivera nos choix pour l'acquisition des données et l'extraction des paramètres caractéristiques de l'écriture.

- Nous décrirons par la suite le protocole d'acquisition, la base de données collectée à ce jour, ainsi que l'ensemble des paramètres extraits pour l'analyse.

- La dernière partie présentera nos conclusions et les perspectives du présent travail.

\section{Etat de l'art et protocole d'acquisition}

\subsection{Analyse de l'écriture manuscrite en ligne}

L'écriture manuscrite est une activité conventionnelle et codifiée qui nécessite un apprentissage, cet apprentissage pouvant être différent d'un pays à un autre, d'une culture à une autre, et 
évidemment d'une langue à une autre. L'écriture peut être dégradée avec l'âge, l'état de santé physique et mental du scripteur et selon le contexte linguistique ou la tâche effectuée : Brouillons, notes personnelles, lettres intimes ou officielles. La trace écrite prend plusieurs aspects différents.

L'analyse de cette trace écrite, s'avère nécessaire et importante pour voir comment cette dernière change ou se dégrade à travers l'âge, les pathologies. D'ailleurs, plusieurs études ont été faites sur l'écriture manuscrite pour élaborer un diagnostic simple et précoce des pathologies neurodégénératives à savoir le Parkinson, l’Alzheimer, et les Troubles Cognitifs Légers.

\subsubsection{La maladie de Parkinson}

La maladie de Parkinson est la seconde maladie neurodégénérative la plus répandue, après la maladie d'Alzheimer. Elle se traduit par une destruction lente et progressive des neurones de la substance noire du cerveau [1]. Il en résulte des symptômes moteurs (tremblements, rigidité musculaire, impossibilité ou ralentissement des mouvements) mais aussi parfois des atteintes intellectuelles (détérioration de la mémoire et difficulté à adapter son comportement au changement de situation) [2]. L'âge moyen de début de la maladie se situe entre 55 et 65 ans, mais il existe des formes précoces qui débutent aux alentours de 40 ans, et des formes tardives qui débutent après 75 ans.

\subsubsection{La maladie d'Alzheimer}

La maladie d'Alzheimer est une affection neurodégénérative qui entraîne une détérioration progressive et définitive des cellules nerveuses provoquant la forme la plus commune des démences. Cette maladie peut affecter tous les aspects de la vie d'une personne, c'est-à-dire ses pensées, ses émotions et son humeur, ainsi que ses comportements et ses habilités physiques.

La maladie d'Alzheimer touche environ 1\% des personnes âgées de 65 ans à 69 ans, 20\% des personnes ayant 85 ans à 89 ans et $40 \%$ des personnes ayant 90 ans à 95 ans. Plus la maladie survient à un âge avancé, plus elle tend à s'aggraver rapidement. Lorsqu'elle se manifeste vers l'âge de 60 ans ou de 65 ans, en moyenne l'espérance de vie est d'environ 12 ans à 14 ans. Lorsqu'elle survient plus tard, l'espérance de vie n'est plus que de 5 ans à 8 ans [3].

La maladie d'Alzheimer n'est pas une conséquence du processus normal du vieillissement. Elle est due à une accumulation de protéines anormales dans le cerveau. On ne connait pas exactement toutes les causes de cette maladie. Actuellement, certains symptômes peuvent être soignés mais il n'existe pas encore de traitement curatif. Parmi les symptômes les plus répandus, on peut citer les troubles de la mémoire, l'aphasie, la bradykinésie, le trouble de l'équilibre, et la dysorthographie [4].

\subsubsection{Etat de l'art}

L'écriture est une composante du langage complexe, apparue tardivement dans l'histoire de l'humanité et qui se développe également tardivement chez l'individu. La détérioration de l'écriture, quelle que soit son importance, est le signe d'un dysfonctionnement d'un programme moteur fin.

Plusieurs études ont été menées sur l'écriture des personnes souffrantes de pathologies, comme une qui avait comme but principal l'analyse de la cinématique de la production d'écriture, menée auprès de 4 participants ayant tous plus de 65 ans, à savoir personnes atteintes d'Alzheimer, 3 personnes ayant des troubles cognitifs légers et 41 personnes en bonne santé. Toutes ces personnes ont été invitées à copier, sur une tablette Wacom, un numéro de téléphone, une liste d'articles d'épicerie (mots), à remplir un chèque, à copier les lettres de l'alphabet hébreu, ainsi qu'un paragraphe de 107 caractères [5].

Pour évaluer la différence entre groupes concernant les caractéristiques sociodémographiques (sexe, âge, nombre d'années d'éducation) et aussi par rapport aux paramètres cinématiques 
(pression, temps du stylet en l'air, temps sur papier, vitesse moyenne, la distance entre le début et la fin de chaque lettre), les auteurs ont utilisé une ANOVA (analyse de variance), ainsi qu'un Test statistique post-hoc de Scheffé pour voir quels sont les groupes qui sont statistiquement différents. Grâce à une analyse discriminante, ils ont trouvé que les MCI et les personnes atteintes d'Alzheimer passent significativement plus de temps en l'air que les personnes saines, et ils exercent également une pression moins forte que les personnes saines. En effet, la pression et la vitesse se sont révélées les plus stables sur toutes les tâches, alors que les autres paramètres temporaires et spatiaux changent avec la complexité de la tâche à effectuer.

Parallèlement, une autre étude a été menée sur 28 patients atteints du Parkinson et 28 personnes saines, à qui on a demandé d'écrire une série de boucles ' $\ell$, et de copier une phrase complète [6]. Cette étude a montré que l'écriture des patients atteints de Parkinson est désordonnée, contrairement à celle des personnes qui prennent des médicaments, le contrôle du tremblement durant l'écriture étant bien meilleur.

Les mouvements en l'air sont aussi très importants. C'est une conclusion d'une étude qui a été faite en République Tchèque où la plupart des paramètres discriminants viennent de l'écriture d'une phrase imposée. Cette tâche donc semble bien prédictive [7].

\subsection{Protocole d'acquisition et matériel utilisé}

L'acquisition des données est en cours depuis mois au sein du service de Neurologie du Centre Hospitalier Universitaire (CHU) Hassan II-Fès. Nous sommes intéressés par la population marocaine répartie par tranche d'âge de [15-25] [25-35] [35-45] [45-55] [55-65] [65-75] [75-85], par les patients atteints des maladies de Parkinson et d'Alzheimer, ainsi que les patients atteints des Troubles Cognitifs Légers (MCI) et qui appartiennent à la même tranche d'âge.

\subsubsection{Critères d'inclusion et de non inclusion}

Pour la sélection des participants, nous avons défini des critères d'inclusion et de non inclusion.

\subsubsection{Critères d'inclusion}

- Accepter de participer librement à l'étude en signant un formulaire de consentement.

- Etre un patient suivi en consultation neurologique au CHU HASSAN II Fès.

- Tous les patients ont passé un bilan cognitif complet leur permettant d'être inclus ou exclus de l'étude. Critères d'inclusion par groupe :

- Groupe 1 : Sujets contrôles ayant un fonctionnement cognitif global normal.

- Groupe 2 : Patients Parkinsoniens, MMSE (Mini Mental State Examination : Echelle mesurant le niveau de dégradation cognitif) entre 20 et 25 , Echelle motrice UPDRS, bilan neuropsychologique complet.

- Groupe 3: Patients atteints d'Alzheimer débutant avec un MMSE entre 20 et 25.

- Groupe 4 : Patients souffrant des Troubles Cognitifs Légers.

\subsubsection{Critères de non-inclusion}

- Patients analphabètes.

- Patients ayant des troubles visuels ou auditifs leur empêchant de réaliser convenablement les tâches.

- Toute personne exclue par le test MMSE pour des raisons comme le traumatisme crânien, AVC, ou autre. 
- Sujets sous régime de protection curatelle ou tutelle.

\subsubsection{Matériel utilisé}

L'acquisition de l'écriture manuscrite se fait sur une tablette graphique

«WACOM Intuos Pro » à stylet créative (figure 1).

Cette tablette est capable de détecter 2048 niveaux de sensibilité à la pression, avec une fréquence d'échantillonnage de $125 \mathrm{HZ}$ permettant d'enregistrer au cours du temps les coordonnées du stylo $(x(t), y(t))$, son inclinaison, ainsi que ses coordonnées lorsqu'il est en l'air (jusqu'à . cm au dessus de la tablette).

Cette tablette ne permet pas un retour visuel du tracé effectué mais permet de capturer les signaux à travers une feuille de papier posée sur elle ; avec un stylo spécial "Inking Pen » de Wacom (stylo à encre), la personne peut avoir un retour visuel du tracé qu'elle effectue sur le papier.

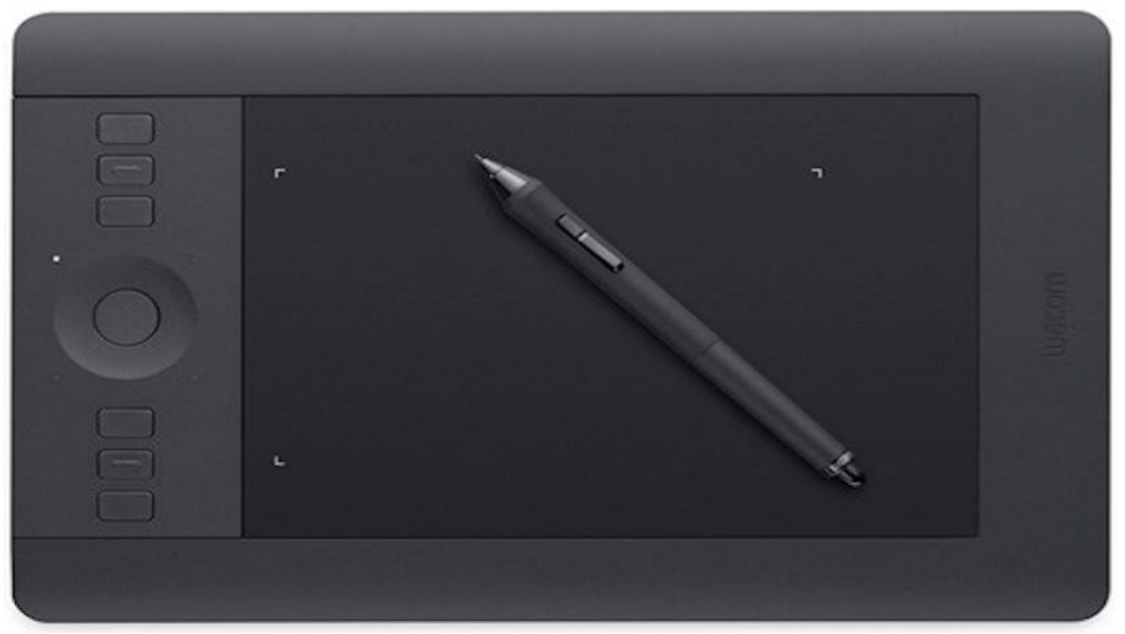

Figure 1. La tablette graphique Wacom Intuos Pro

\subsubsection{Protocole d'acquisition}

L'acquisition des données est en cours depuis 10 mois au sein du service de Neurologie du Centre Hospitalier Universitaire Hassan II-Fès.

Les participants volontaires sont invités à reproduire des écrits selon un protocole prédéfini. Les données sont recueillies de façon anonyme et confidentielle, et tous les participants ont le droit de se retirer de l'étude à n'importe quel moment.

Nous commençons par saisir les métadonnées concernant le participant sur l'interface d'un logiciel lié à la tablette. L'évaluation complète d'un patient dure une heure et trente minutes : Une heure pour le bilan neuropsychologique et trente minutes pour l'acquisition de l'écriture. Pour les sujets contrôles, nous leur faisons passer un bilan de 20 min afin de connaître leur profil cognitif pour s'assurer, entre autres, qu'ils ont un profil cognitif normal.

Tous les participants devaient réaliser, dans l'ordre, un certain nombre de tâches, que nous leur avons présentées au fur et à mesure. Notre étude se compose de 3 exercices concernant 1'Arabe, 3 exercices concernant le Français, et finalement 4 exercices de dessins. Le premier exercice consiste à recopier un texte imposé en Arabe et en Français (figure 2). Le deuxième exercice consiste à écrire un texte libre en Arabe et un texte libre en Français, suivis d'une série de quatre lettres " $\ell$ 》 cursives reliées entre elles à écrire quatre fois pour le Français, et une série de 4 lettres « ص reliées entre elles, quatre fois aussi pour l'Arabe. Le reste des exercices de dessins consiste à dessiner des 
cercles, une spirale, des allers-retours entre deux carreaux. Le modèle de la feuille d'acquisition est représenté dans la figure suivante :

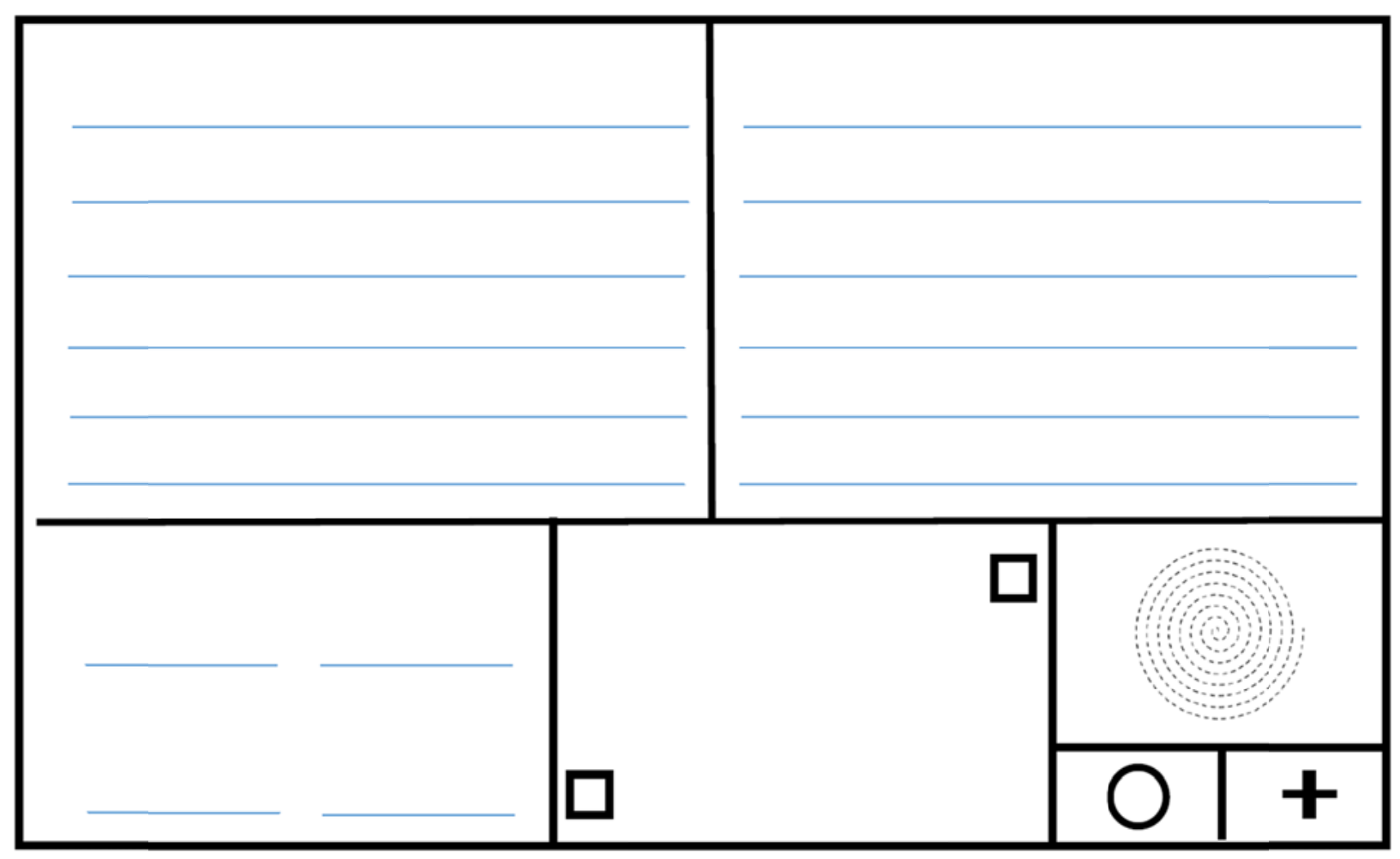

Figure 2. Modèle général de la feuille d'acquisition

\subsection{Base de données acquise au CHU Fès}

A ce jour, nous avons pu acquérir les données auprès de 122 personnes qui sont réparties comme suit : 24 Parkinsoniens, 2 MCI, 2 Alzheimer, et 94 personnes contrôles. Notre base de données est constituée de 58 femmes et 64 hommes, dont 118 sont des droitiers et 4 sont des gauchers. Le niveau d'études, la profession, la fréquence d'écriture et la tranche d'âge sont des facteurs qui seront pris en considération lors de l'analyse.

En principe, tous les participants seront revus deux fois. La première visite a lieu au mois M0 et la seconde au mois M12 (12 mois plus tard) pour réacquérir leurs données avec le même protocole et refaire un bilan neuropsychologique.

\subsection{Extraction des paramètres descriptifs}

Pour chaque point de la trajectoire du stylet, nous avons les paramètres $(x(n), y(n), P(n))$ qui représentent respectivement l'abscisse, l'ordonnée et la pression du stylet. Les paramètres extraits peuvent être classés selon 3 catégories :

- Cinématiques : vitesse (en $\mathrm{x}, \mathrm{y}$, et le module), accélération, jerk ou dérivée de l'accélération, etc.

- Spatiaux : Direction, courbure, longueur des espaces intra-mots, longueur des espaces intermots, hauteur des mots, complexité de la trajectoire du stylo (au moment où le stylo est en l'air) ...

- Dynamiques : Pression, variation de pression.

Dans la catégorie de paramètres cinématiques, les vitesses horizontale et verticale sont estimées en chaque point $n$ de la trajectoire du stylet de la façon suivante

$$
\mathrm{Vx}(\mathrm{n})=\Delta \mathrm{x}(\mathrm{n}) / \Delta \mathrm{t}(\mathrm{n}) \text { et } \mathrm{Vy}(\mathrm{n})=\Delta \mathrm{y}(\mathrm{n}) / \Delta \mathrm{t}(\mathrm{n})
$$


$\Delta x(n)=x(n+1)-x(n-1)$,

$\Delta \mathrm{y}(\mathrm{n})=\mathrm{y}(\mathrm{n}+1)-\mathrm{y}(\mathrm{n}-1)$

$\Delta \mathrm{t}(\mathrm{n})=\mathrm{t}(\mathrm{n}+1)-\mathrm{t}(\mathrm{n}-1)$.

\subsection{Visualisation des données}

Afin d'illustrer ces informations locales, nous présentons en figure 3 et 4 un texte écrit par un participant montrant respectivement toutes les valeurs des vitesses en $\mathrm{Y}$ (Vy) et de pression de l'écriture par un code couleur.
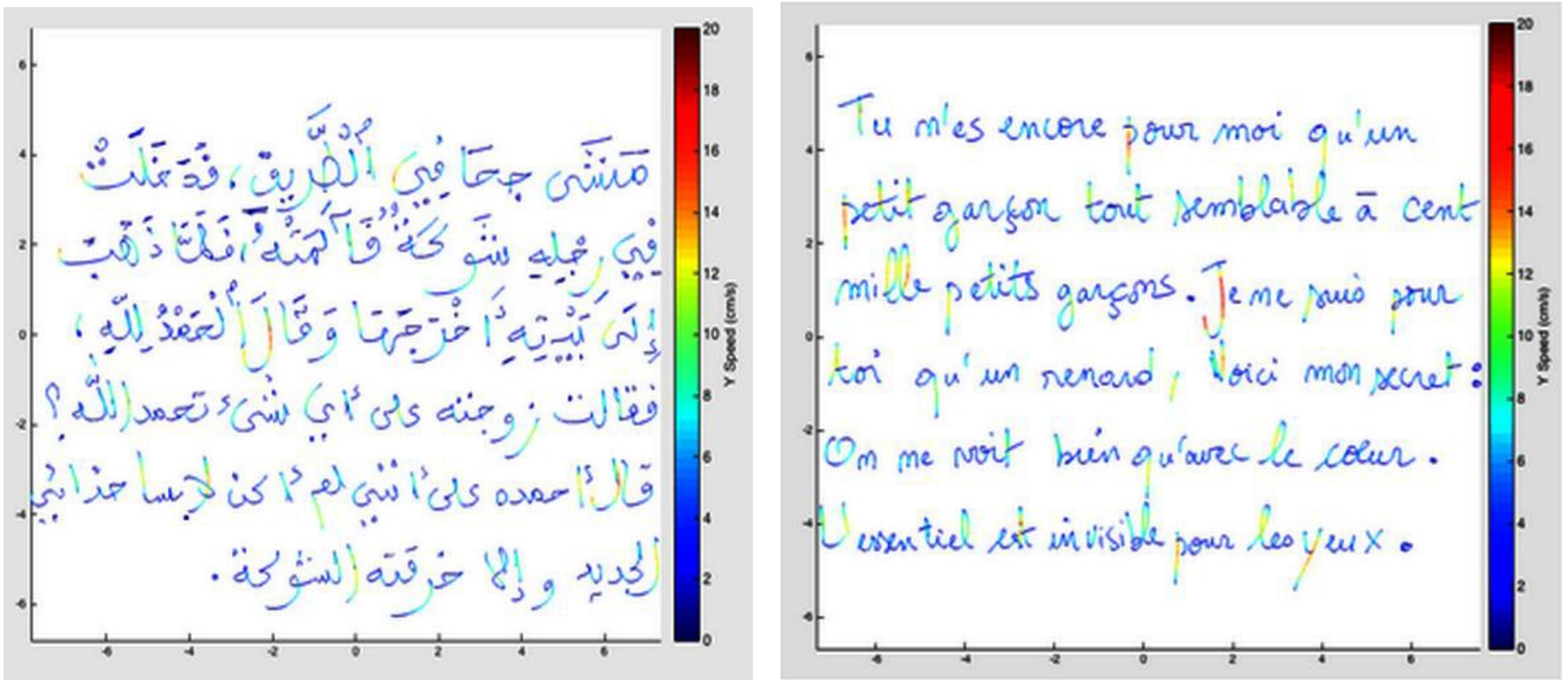

Figure 3. Visualisation de la vitesse en $Y(V y)$

La couleur bleue représente les vitesses faibles et la couleur rouge représente les vitesses fortes sur des plages allant de 0 à $20 \mathrm{~cm} / \mathrm{s}$.
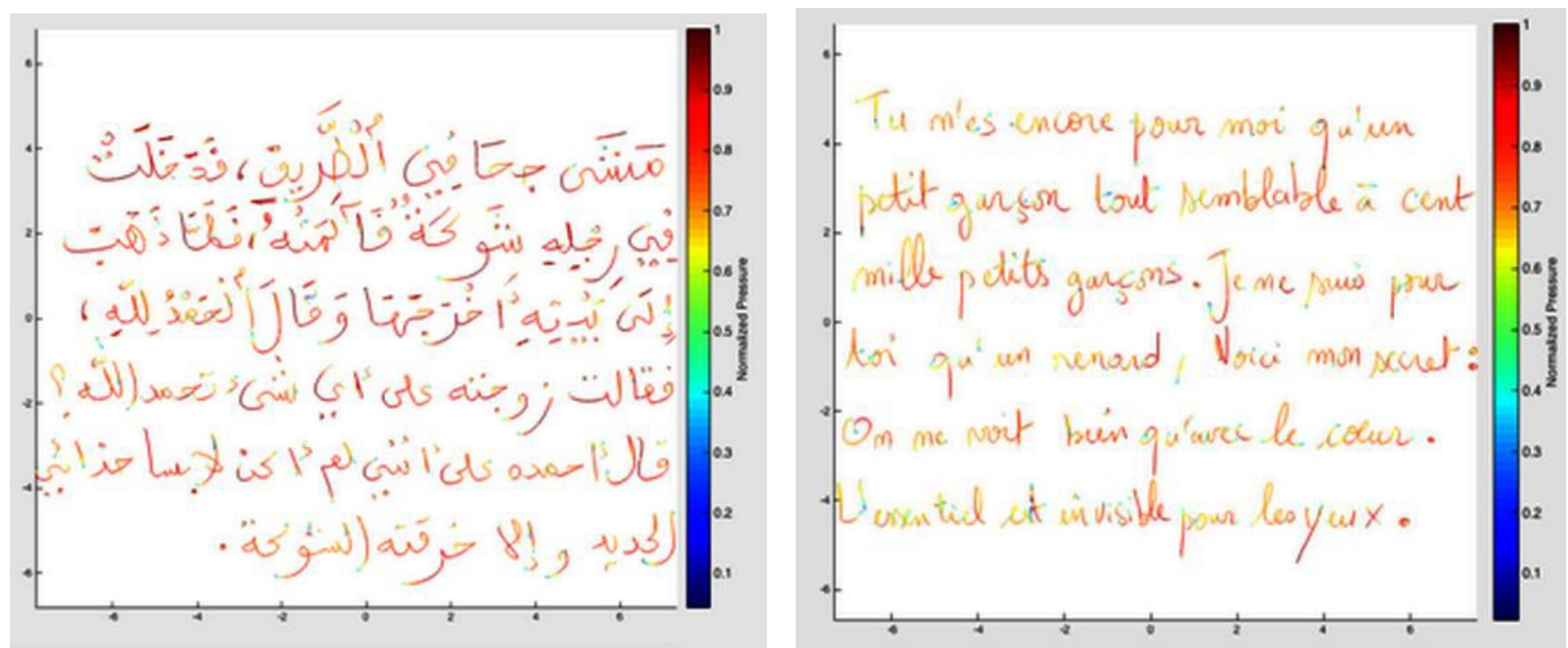

Figure 4. Visualisation de la pression d'écriture

La couleur bleue représente les pressions faibles et la couleur rouge représente les pressions fortes sur des plages allant de 0 à 1 . 


\section{Conclusion et Perspectives}

L'analyse automatique de l'écriture manuscrite est une approche complémentaire à d'autres approches visant à déterminer les points les plus discriminants entre les personnes normales et les patients atteints d'une pathologie neurodégénérative, notamment, le Parkinson, l'Alzheimer ou MCI. Notons qu'il existe des études récemment élaborées qui font principalement l'analyse de la voix ou de la démarche et qui ont en principe les mêmes objectifs que notre étude. Généralement, les travaux scientifiques qui cherchent à identifier un groupe de facteurs discriminants entre les patients et personnes normales pourront connaitre des succès scientifiques importants.

Concernant cette étude, nous envisageons, sur le plan des acquisitions de l'écriture, d'enrichir notre base de données avec des personnes plus âgées pour pouvoir étudier l'impact de l'âge sur la dégradation de l'écriture manuscrite, et distinguer ainsi les effets d'un vieillissement normal de ceux dus à un état pathologique. Nous ciblerons aussi le recrutement des sujets atteints de schizophrénie afin d'évaluer la possibilité de dégradation de l'écriture par cette maladie.

Sur le plan pratique, nous visons entamer une étude statistique une fois que nous aurons une base de données homogène et représentative, en se basant sur tout ce qui a été abordé dans la littérature et en cherchant de nouveaux paramètres discriminants capables de porter une valeur diagnostique pour ces pathologies.

\section{Bibliographie}

[1] ANNE-MARIE BONNET, THIERRY HERGUETA. « La maladie de parkinson au jour le jour», A33.

[2] AWA Van Gemmert, C Adler, and G Stelmach. "Parkinson's disease patients undershoot target size in handwriting and similar tasks », J Neurol Neurosurg Psychiatry, n 74, p. 1502-1508, 2003.

[3] O.ENGASSER «L'atelier d'écriture : Une indication pour diminuer les troubles du comportement dans la maladie d'Alzheimer et maladie apparentées au stade évolué».

[4] A. Schroter, R. Mergl, K. Burger, H. Hampel, H.-J. Moller, U. Hegerl. « Kinematic Analysis of Handwriting Movements in Patients with Alz- heimer's Disease, Mild Cognitive Impairment, Depression and Healthy Subjects», Dementia and Geriatric Cognitive Disorders - DEMENT GE- RIATR COGN DISORD,2003, vol. 15, no. 3, pages. 132-142.

[5] I. Werner, P., Rosenblum, S., BAR-On, G., HeiniK, J., KorCZyn, A. (2006)., « Handwriting process variables discriminating mild Alzheimer's disease and mild cognitive impairment ». The Journals of Gerontology Series B: Psychological Sciences and Social Sciences, 61(4), P228-P236.

[6] Atilla unlu, Rudiger Brause, Karsten Krakow, « Handwriting Analysis for Diagnosis and Prognosis of Parkinson's Disease».

[7] P. Drotar, J. Mekyska, I. Rektorova, L. Masarova, Z. Smekal, M. Faundez-Zanuy., « A new modality for quantitative evaluation of Parkin- son's disease : In-air movement ", IEEE 13th International Conference on Bioinformatics and Bioengineering (BIBE), 2013. 